\title{
From Brownian motion to power of fluctuations
}

The year 2012 marks the 140th birth anniversary of Marian Smoluchowski (28.05.1872-5.09.1917), a man who "made ground-breaking contribution to the theory of Brownian motion, the theory of sedimentation, the statistical nature of the Second Law, the theory and practice of density fluctuations (critical opalescence). During his final years of scientific creativity his pioneering theory of coagulation and diffusion-limited reaction rate appeared. These outstanding achievements present true gems which dominate the description of soft matter physics and chemical physics as well as the related areas up till now!” This quotation was taken from the lecture by Peter Hänggi given at international conference Statistical Physics: Modern Trends and Applications that took place in Lviv, Ukraine on July 3-6, 2012 (see http://www.icmp.lviv.ua/statphys2012/ for more details) and was dedicated to the commemoration of Smoluchowski's work. This and forthcoming issues of the Condensed Matter Physics contain papers presented at this conference.

Marian Smoluchowski's (Maryan Ritter von Smolan Smoluchowski) life was bright but, unfortunately, ended up far too early. Being Polish by origin, Marian Smoluchowski was born in Vienna. Here he was growing up, studying and in 1895 received his doctoral degree from the University of Vienna. At the age of forty five Marian Smoluchowski unexpectedly died in Kraków, shortly after moving there from Lviv, where he spent his most fruitful fourteen years from 1899 till 1913, all this time working for the Lviv University. A detailed study of this period of Marian Smoluchowski's life is presented in the article by Andrij Rovenchak that follows this preface note. Besides other details, this paper presents a complete list of papers by Smoluchowski that refer to the Lviv period of his life. Among this list one will find his keystone papers on the temperature jump at the surface of a solid body, on the theory of fluctuations and the theory of Brownian motion, on kinetic theory of colloid coagulation and electrokinetic phenomena.

This special issue of Condensed Matter Physics, entitled From Brownian motion to power of fluctuations, is composed in total of eighteen papers presented at the conference, and in conjunction with one of European Physical Journal Special Topics, entitled From Brownian motion to self-avoiding walks and Lévy flights, are aimed at reflecting the evolution of Smoluchowski's ideas from the field of statistics of interacting random and self-avoiding walks, stochastic equations for many-particle systems, physics of glass-forming and noise driven systems to the theory of fluctuations with the particular emphasis on soft matter and systems with long-range order.

Among eighteen papers of this issue there are two - the above mentioned paper by Rovenchak and another one by Ilnytska et al. - that are dedicated to shed more light on the Lviv period of Marian Smoluchovski and, we believe, could be helpful to those interested in the history of science and physics in particular.

The remaining sixteen papers can be divided into groups. The first one includes papers devoted to the particular mathematical issues of the theory of condensed mater, including the exactly solvable models. Namely, by using the structure of 29-dimensional extended real Clifford-Dirac algebra, in their paper Simulik et al. try to prove the property of Fermi-Bose duality of the Dirac equation with nonzero mass. It means that Dirac equation can describe not only the fermionic but also the bosonic states. On other hand, Morozov and Ropke present analytical results for the time-dependent information entropy in exactly solvable two-state (qubit) models, while Shvaika and Freericks examine the core-level X-ray photoemission spectroscopy, X-ray absorption near-edge spectroscopy and X-ray emission spectroscopy in the FalicovKimball model by using the exact solution from dynamical mean-field theory. Two other papers, one by Strečka and Ekiz and the second by Strečka et al., present and discuss the exact solutions for the spin-1/2 Ising-Heisenberg model on diamond-like decorated Bethe lattices and for the ferrimagnetic spin-1/2 chain composed of alternating Ising and Heisenberg spins in an arbitrarily oriented magnetic field, respectively. 
Modern tools of statistical physics of condensed matter such as computer simulations and density functional theory and their applicability to particular problems are considered in the second group of papers. Kaupužs et. al present the Monte Carlo analysis of the Goldstone mode singularities for transverse and longitudinal correlation functions performed in the three-dimensional $O(n)$ models with $n=2,4,10$. Druchok and Holovko have applied the all-atom molecular dynamics simulations to explore an association behavior of uranyl ions in aqueous solutions. Huerta et al. discuss the freezing transition in a twodimensional hard-core matter and its frustration by going to the equimolar binary mixtures. Marenz et al. report the results of the Monte Carlo simulations on investigation of the effect of a spherical confinement within a simple model for a flexible homopolymer. Finally, Schmidt et al. have outlined an overview of several recent developments in density functional theory for classical inhomogeneous liquids.

The third group of papers includes those that consider various aspects of the problem of phase transitions in the condensed matter. Dudka et al. discuss the conditions for realization of different types of multicritical behaviour within the field-theoretical renormalization group approach. In the paper by Pylyuk and Ulyak, the critical behaviour of systems belonging to the three-dimensional Ising universality class is studied theoretically using the collective variables method.

Finally, there is a group of papers where statistical mechanics approaches are applied to particular condensed matter systems. In the paper by Sokolovsky, the Chapman-Enskog method is generalized for the purpose of accounting the effect of kinetic modes on hydrodynamic evolution and for simplicity, the investigation was carried out for crystals of the cubic class symmetry. Khodusov and Naumovets propose a possible mechanism of interaction of microwaves with superfluid helium that results in an experimentally observed narrow peak of microwave absorption upon the frequencies by the order of the roton frequency. Derzhko et al. consider the antiferromagnetic Heisenberg model on a distorted diamond chain and use the localized-magnon picture adapted to a distorted geometry to discuss some of its highfield low-temperature properties. Gomonay and Loktev analyse the statistical properties of the currentdriven AFM nanooscillator that result from the white Gaussian noise of magnetic nature. Concerning this last paper, spin-torque effects in antiferromagnetic materials are of great interest due to the possibility of being applied as high-speed spintronic devices.

We express our appreciation to all the authors for their commitment. It is also a pleasure to thank Yaroslav Shchur and Orest Tsurkovskii for the assistance in editing the papers.

Bertrand Berche (Nancy) Myroslav Holovko (Lviv) Andrij Trokhymchuk (Lviv) Vojko Vlachy (Ljubliana) 